\title{
Extraction and Reglucosylation of Barbarea vulgaris Sapogenins
}

Jörg M. Augustin ${ }^{1^{\star}}$, Carl Erik Olsen ${ }^{2}$ and Søren Bak²

${ }^{1}$ Faculty of Life Sciences - Department for Plant Biochemistry and Biotechnology, University of Copenhagen, Fredriksberg, Copenhagen, Denmark; ${ }^{2}$ Faculty of Life Sciences - Department of Basic Science and Environment, University of Copenhagen, Fredriksberg, Copenhagen, Denmark

*For correspondence: imaug@gmx.de

[Abstract] Plants produce a vast array of natural compounds. Many of them are not commercially available, and are thus lacking to be tested as substrates for enzymes. This protocol describes the extraction and acidic hydrolysis of metabolites from Barbarea vulgaris with special focus on saponins and their agylcones (sapogenins). It was developed to determine if some $B$. vulgaris UDP-glucosyltransferases (UGTs) that were shown to glucosylate commercially available sapogenins, would also accept additional sapogenins from this plant as substrate, which are yet chemically uncharacterized and/or commercially unavailable (Figure 1).

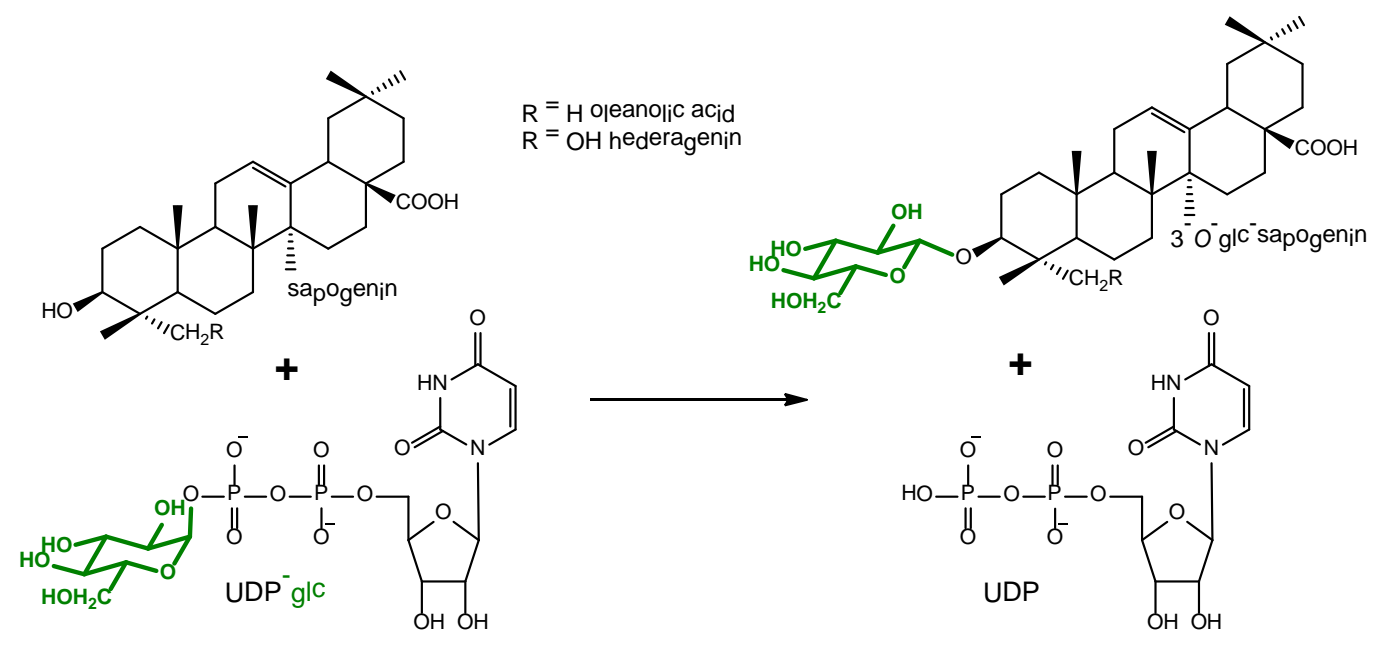

Figure 1. Glucosylation reaction catalyzed by UGT73C10-UGT73C13 from Barbarea vulgaris (Augustin et al., 2012). All four enzymes utilize uridine diphosphate glucose (UDPglc) as glucosyl-moiety donor and different sapogenins such as the oleanane sapogenins oleanolic acid and hederagenin as glucosyl-moiety acceptor. Oleanolic acid and hederagenin both naturally occur in G-type B. vulgaris, where they are predominantly found in their 3-Ocellobiosylated form. Additional saponins from G-type $B$. vulgaris have been identified by Nielsen et al. (2010). However, the majority of saponins and sapogenins that occur in $B$. vulgaris remain unidentified. 


\section{Materials and Reagents}

1. Bovine serum albumin (BSA) (Sigma-Aldrich, catalog number: A7906)

2. Polyvinylpolypyrrolidone (PVPP) (Sigma-Aldrich, catalog number: 77627)

3. Hydrochloric acid $(\mathrm{HCl})$ (Sigma-Aldrich, catalog number: $\mathrm{H} 1758)$

4. Tris(hydroxymethyl) aminomethane (Tris base) (Sigma-Aldrich, catalog number: T1503)

5. Ethyl acetate (Sigmal-Aldrich, catalog number: 34972)

6. N-Tris(hydroxymethyl)methyl-3-aminopropanesulfonic acid (TAPS) (Sigma-Aldrich, catalog number: T5130)

7. Dithiothreitol (DTT) (Sigma-Aldrich, catalog number: D0632)

8. Uridine-5'-diphosphoglucose (UDP-Glc) (Sigma-Aldrich, catalog number: S451649)

9. Silica gel $60 F_{254}$ TLC plates (EMD Millipore, catalog number: 1055540001)

10. Polyvinylidene difluoride (PVDF) filter plate $(0.45 \mu \mathrm{m}$ pore diameter) (EMD Millipore, catalog number: MAHVN4510)

11. FRETWorks S-tag assay kit (EMD Millipore, catalog number: 70724)

\section{Equipment}

1. Water bath

2. Centrifuge for $50 \mathrm{ml}$ and $15 \mathrm{ml}$ conical centrifugation tubes (VWR international, catalog number: 89004-368)

3. Thermomixer (VWR international, catalog number: 21516-168)

4. $\mathrm{pH}$ indicator paper (Whatman, catalog number: 2600-100A)

5. Vacuum centrifuge (Labogene, catalog number: 7.008.100.777)

6. Thin layer chromatography (TLC) developing chamber (VWR international, catalog number: 21432-739)

7. Aldrich flask-type sprayer (Sigma-Aldrich, catalog number: Z190373)

8. Heat block (VWR international, catalog number: 12621-120)

9. LC-MS analysis was carried out on an Agilent 1100 Series LC (Agilent Technologies), equipped with a Gemini NX column (Phenomenex), and coupled to a Bruker HCT-Ultra ion trap mass spectrometer (Bruker Daltonics)

\section{Software}

1. DataAnalysis 4.0 (Bruker Daltonics) 


\section{Procedure}

A. Preparation of the crude metabolite extract:

1. Freshly harvested Barbarea vulgaris leaves were weighed and transferred to $15 \mathrm{ml}$ centrifugation tubes.

2. Following addition of $5 \mathrm{ml} 55 \%$ ethanol per $\mathrm{g}$ fresh leaf material the leaves were boiled in a water bath for $10 \mathrm{~min}$.

3. To increase the extraction efficiency, the tubes were occasionally shaken while boiling.

4. After heating the extracts were chilled on ice before they were centrifuged for $5 \mathrm{~min}$ $(3,000 \times g$, room temperature) to precipitate insoluble leaf debris.

5. The supernatant was transferred to fresh centrifugation tubes and stored at $-20{ }^{\circ} \mathrm{C}$ until further usage. A minimum incubation time of $4 \mathrm{~h}$ at $-20^{\circ} \mathrm{C}$ is recommended to cause further unwanted compounds to precipitate from the solution.

6. Newly emerged precipitates were removed by centrifugation $\left(3,000 \times g, 5 \mathrm{~min}, 4^{\circ} \mathrm{C}\right)$. Notes:

a. Usage of the protocol has been limited so far to rosette leaves of 1-3 month old Barbarea vulgaris plants with a typical weight of 1.5-2 $\mathrm{g}$ fresh weight.

b. Saponins can be extracted with this protocol from both fresh and ground plant material.

c. $55 \%$ ethanol has been determined in pre-experiments to be hydrophobic enough to still extract $B$. vulgaris saponins, while being hydrophilic enough to lower the amount of some hydrophobic compounds that were previously seen to interfere with TLC analysis. However, it should be noted that these extracts still contains many more compounds than just saponins.

B. Acidic hydrolysis and purification:

1. $2 \times 1.25 \mathrm{ml}$ of the crude saponin extract were transferred into $2 \mathrm{ml}$ microcentrifuge tubes and mixed with $250 \mu \mathrm{l} 6 \mathrm{M} \mathrm{HCl}$ to adjust the final $\mathrm{HCl}$ concentration to $1 \mathrm{M}$.

2. The acidified extracts were incubated for $24 \mathrm{~h}$ in a thermomixer adjusted to a temperature of $99^{\circ} \mathrm{C}$ and shaking at $1,400 \mathrm{rpm}$.

3. After heating the extracts were chilled for approximately $1 \mathrm{~h}$ at $-20{ }^{\circ} \mathrm{C}$ before they were combined in $50 \mathrm{ml}$ centrifugation tubes.

4. Remaining precipitates in both microcentrifuge tubes were recovered by washing each tube three times with $250 \mu \mathrm{l} 96 \%$ ethanol. The resulting ethanol solutions of these three wash steps were added to the hydrolysate in the $50 \mathrm{ml}$ centrifugation tubes.

5. $1 \mathrm{M}$ Tris base solution was added to the hydrolysate until the $\mathrm{pH}$ shifted from acidic to basic conditions (here: $4.5 \mathrm{ml}$ ). 
6. Subsequently, $13.55 \mathrm{ml}$ water was added to lower the ethanol concentration to $14 \%$. $1.125 \mathrm{~g}$ PVPP and $225 \mathrm{mg}$ BSA were added to the solution to adjust their final concentrations to $5 \%(\mathrm{w} / \mathrm{v})$ and $10 \mathrm{mg} / \mathrm{ml}$, respectively.

7. The mixture was six times extracted ethyl acetate using $5 \mathrm{ml}$ ethyl acetate per extraction step.

8. Phase separation was achieved by centrifugation for $20 \mathrm{~min}$ at $5,200 \times \mathrm{g}$. The ethyl acetate fraction will be the upper phase.

9. The combined ethyl acetate fractions were evaporated to dryness in a vacuum centrifuge.

10. Dried extracts were dissolved in $500 \mu \mathrm{l} 96 \%$ ethanol and transferred to $15 \mathrm{ml}$ centrifugation tubes. For a second round of purification 3,720 $\mu$ water, $480 \mu \mathrm{l} 500 \mathrm{mM}$ TAPS pH 9.1, $240 \mathrm{mg}$ PVPP and 48 mg BSA were added in the given order and 5-fold ethyl acetate extraction performed with $2 \mathrm{ml}$ ethyl acetate per extraction step.

11. After evaporation of the solvent of the combined ethyl acetate fractions in a vacuum centrifuge, the dried extracts were dissolved in $1 \mathrm{ml} \mathrm{96 \%} \mathrm{ethanol.}$

Notes:

a. Brief spinning in a tabletop microcentrifuge was found sufficient during the washing steps to recover precipitates from the hydrolysate.

b. Due to a lack of investigations if sapogenins will remain solubilized in the chosen hydrolysation conditions or are among the observed precipitates both fractions combined were subjected to subsequent purification steps.

c. The $\mathrm{pH}$ of the hydrolysate was shifted to basic conditions by addition of Tris base prior extraction, since ethyl acetate extraction carries over low amounts of water/ions, which caused the initial hydrolysate extracts to be of slightly acidic $\mathrm{pH}$. The UGTS investigated by us had a slightly basic $\mathrm{pH}$ optimum and a weakly basically buffered sapogenin extract was considered to have a lower effect on the $\mathrm{pH}$ of the final enzyme assay.

d. $\mathrm{pH}$ changes were monitored by spotting $1 \mu \mathrm{l}$ of the hydrolysate to $\mathrm{pH}$ indicator paper.

e. The ethanol concentration of the hydrolysate had to be lowered prior ethyl acetate, extraction to enable formation of an organic phase upon addition of ethyl acetate.

f. Early ethyl acetate extracts of hydrolysated crude Barbarea vulgaris leaf extracts generated without the PVPP/BSA purification step were seen to completely inhibit the activity of the investigated UGTs. PVPP was used to adsorb phenolic compounds. BSA was added in the purification step, since in enzyme assays using the early hydrolysation extracts proteins were seen to become brownish by binding to compounds from the extract. The addition of BSA was intended to remove such protein binding compounds. 
g. While drying down the ethyl acetate fractions in the vacuum centrifuge, new aqueous phases emerged, which were removed in the process.

C. Re-glucosylation assay:

1. In preparation of the re-glucosylation assays $500 \mu \mathrm{l}$ of the hydrolysated and purified $B$. vulgaris leaf metabolite extracts were dried out in a vacuum centrifuge and subsequently dissolved in $78.13 \mu \mathrm{l}$ dimethyl sulfoxide (DMSO).

2. Additionally, the recombinant expressed UGTs were directly quantified within E. coli lysates applying the FRETWorks S-tag assay kit.

3. Following quantification, UGT concentrations were adjusted to $50 \mathrm{ng} / \mu \mathrm{l}$ by diluting the $E$. coli lysates with $10 \mathrm{mg} / \mathrm{ml}$ BSA in $10 \mathrm{mM}$ TAPS pH 8.0 .

4. Enzymatic activity assays were performed in $1.5 \mathrm{ml}$ microcentrifugation tubes in a final volume of $50 \mu \mathrm{l}$.

5. Reaction conditions were adjusted to $25 \mathrm{mM}$ TAPS pH 8.6 (UGT73C9-C11), pH 7.9 (UGT73C12/C13) or pH 8.2 (combination of UGT73C9, UGT73C10 or UGT73C11 with UGT73C12 or UGT73C13), $1 \mathrm{mM}$ DTT and $1 \mathrm{mM}$ UDP-Glc. The final UGT amount per reaction was $750 \mathrm{ng}$.

6. Reactions were preincubated for $3 \mathrm{~min}$ at $30{ }^{\circ} \mathrm{C}$ and started by addition of $3.13 \mu \mathrm{l}$ hydrolysated and purified $B$. vulgaris leaf metabolite extract in DMSO.

7. The assays were incubated for 30 (LC-MS only) or 120 (TLC and LC-MS) min at $30{ }^{\circ} \mathrm{C}$, and stopped by addition of $325 \mu \mathrm{l}$ ice cold methanol (LC-MS) or $50 \mu \mathrm{l}$ ice cold ethyl acetate (TLC).

Notes:

a. The solvent of the hydrolysated extracts was exchanged from ethanol to DMSO prior to the re-glucosylation assays, as ethanol was found to act as substrate for the applied UGTs itself.

b. Quantification with the FRETWorks S-tag assay kit is based on regeneration of RNase $S$ activity due the interaction of the $S$ protein (included in the kit) and the $S$ tag N-terminally fused to the recombinant expressed UGTS.

c. The E. coli lysates were diluted with a BSA solution instead of pure buffer, since the recombinant UGTS were seen to lose specific activity upon reduction of the total protein concentration.

d. Whenever combinations of different UGTs were tested, the individual enzymes were applied in equimolar amounts.

D. Analysis by thin layer chromatography (TLC) 
1. Stopped enzymatic reactions were three times extracted with ethyl acetate $(50 \mu \mathrm{l}, 185 \mu \mathrm{l}$ and $50 \mu \mathrm{l})$ :

a. Ethyl acetate was added to the enzymatic reaction and the sample thoroughly mixed for approximately 10-20 sec with a vortex shake (the ethyl acetate added to stop the reaction is at the same time also used for the first extraction step).

b. The samples were centrifuged for $5 \min (16,100 \times g$, room temperature) to achieve phase separation. The ethyl acetate fraction will be the upper phase.

c. The combined ethyl acetate fractions were evaporated to dryness in a vacuum centrifuge and the dried extracts dissolved in $20 \mu \mathrm{l} 96 \%$ ethanol.

d. The re-dissolved extracts were stepwise, completely (3.5 $\mu \mathrm{l}$ per step) loaded to a silica gel TLC plate.

e. TLC plates were pre-run in $100 \%$ methanol until the solvent front was approximately $1 \mathrm{~cm}$ above the loading line.

f. The methanol was left to evaporate in a fume hood, and the TLC plates were subsequently developed using dichloromethane: methanol: water (80:19:1) as mobile phase.

g. Sapogenins and sapogenin-glucosides were visualized by spraying TLC plates with $10 \%$ sulfuric acid in methanol using a flask-type sprayer (or similar) and subsequent heating to $100^{\circ} \mathrm{C}$ on a heat block (Figure 2).

(A) visible light

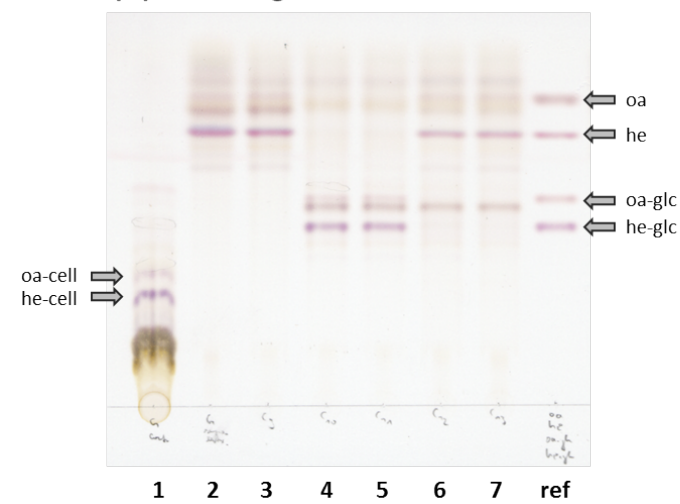

(B) UV $(366 \mathrm{~nm})$

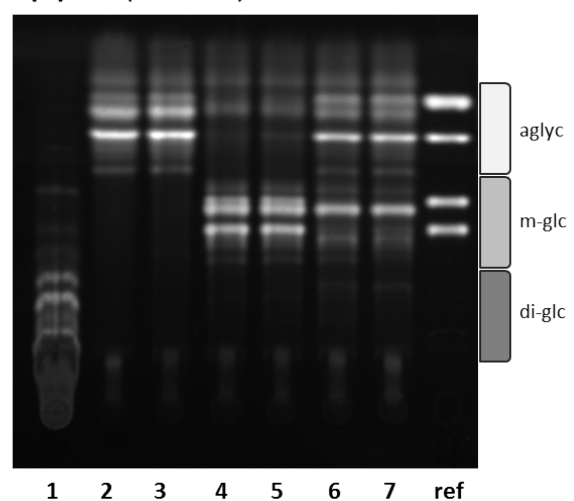

Figure 2. TLC plate with the (1) G-type B. vulgaris crude metabolite extract, the (2) corresponding acidic hydrolyzed metabolite extract and the (3)-(7) hydrolyzed metabolite extract treaded with different $B$. vulgaris UGTs. The TLC plate was evaluated under (A) visible (colored) as well as under (B) long wave UV (366 nm, black/white). The applied UGTs for the reglucosylation assays were (3) UGT73C9, (4) UGT73C10, (5) UGT73C11, (6) UGT73C12, (7) UGT73C13. For comparison purpose were authentic (oa) oleanolic acid, (he) hederagenin, (oa-glc) 3-O-glc oleanolic acid, (he- 
glc) 3-O-glc-hederagenin loaded to the (ref) reference lane (2 nmol each). Additionally are (oa-cell) oleanolic acid cellobioside and (he-cell) hederagenin cellobioside, the naturally in G-type B. vulgaris occurring di-glucosidic forms of these two sapogenins, marked in the crude metabolite extract. The accordingly estimated migration rate of (agly) aglycones, (m-glc) mono-glucosides and (di-glc) di-glucosides are shown on the right of Figure 2B.

Note: The amount of developing solution needed depends on the size of the used TLC plate. The plate should be consistently and homogeneously wetted. However, spraying of too much developing solution may cause the bands to diffuse.

2. Analysis by liquid chromatography-mass spectrometry (LC-MS)

a. Stopped enzymatic reactions were centrifuged for $5 \min (16,100 \times g, R T)$ to precipitate proteins.

b. Supernatants were transferred to fresh $1.5 \mathrm{ml}$ microcentrifugation tubes and evaporated to dryness in a vacuum centrifuge.

c. Dried extracts were dissolved in $30 \mu \mathrm{l}$ methanol and the solvent subsequently diluted to a final concentration of $50 \%$ methanol by addition of $30 \mu$ water.

d. The methanol extracts were filtered (PVDF, $0.45 \mu \mathrm{m}$ pore diameter) and transferred to $1.5 \mathrm{ml}$ glass sample vials for LC-MS analysis.

e. LC-MS analysis was carried out on an Agilent 1100 Series LC, equipped with a Gemini NX column $\left(35^{\circ} \mathrm{C}\right)(2.0 \times 150 \mathrm{~mm}, 3.5 \mu \mathrm{m})$, and coupled to a Bruker HCTUltra ion trap mass spectrometer.

f. Mobile phases in the LC were water with $0.1 \%(\mathrm{v} / \mathrm{V})$ formic acid (eluent $A$ ) and acetonitrile with $0.1 \%(\mathrm{v} / \mathrm{V})$ formic acid (eluent $\mathrm{B}$ ). The gradient program was as follows: 0 to $1 \mathrm{~min}$, isocratic $12 \% \mathrm{~B} ; 1$ to $33 \mathrm{~min}$, linear gradient 12 to $80 \%$ B; 33 to 35 min, linear gradient 80 to $99 \%$ B; 35 to 38 min isocratic $99 \%$ B; 38 to 45 min isocratic $12 \% \mathrm{~B}$ at a constant flow rate of $0.2 \mathrm{ml} / \mathrm{min}$.

g. The MS detector was operated in negative electrospray mode, and $\mathrm{MS}^{2}$ (= MS/MS) and $\mathrm{MS}^{3}$ (=MS/MS of $\mathrm{MS}^{2}$ fragments) fragmentations were performed to obtain additional structural information of the detected ions.

h. Run files were analyzed with Data Analysis 4.0, a software to display the LC chromatograms and the corresponding MS spectrums. Please refer to Augustin et al., 2012 (and Online Supplemental Data) to see the LC chromatograms of crude metabolite extracts from $G$ - and P-type $B$. vulgaris, the acidic hydrolyzed metabolite extracts from both plants as well as chromatograms of the corresponding reglucosylation assays with different $B$. vulgaris UGTs. 


\section{References}

1. Augustin, J. M., Drok, S., Shinoda, T., Sanmiya, K., Nielsen, J. K., Khakimov, B., Olsen, C. E., Hansen, E. H., Kuzina, V., Ekstrom, C. T., Hauser, T. and Bak, S. (2012). UDPglycosyltransferases from the UGT73C subfamily in Barbarea vulgaris catalyze sapogenin 3-O-glucosylation in saponin-mediated insect resistance. Plant Physiol 160(4): 1881-1895.

2. Nielsen, N. J., Nielsen, J. and Staerk, D. (2010). New resistance-correlated saponins from the insect-resistant crucifer Barbarea vulgaris. J Agric Food Chem 58(9): 5509-5514. 